\title{
FOXM1 repression increases mitotic death upon antimitotic chemotherapy through $B M F$ upregulation
}

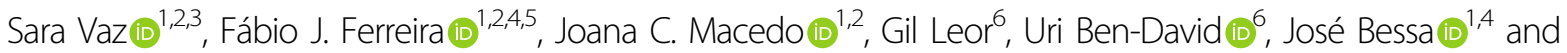 \\ Elsa Logarinho (1) ${ }^{1,2}$
}

\begin{abstract}
Inhibition of spindle microtubule (MT) dynamics has been effectively used in cancer treatment. Although the mechanisms by which MT poisons elicit mitotic arrest are fairly understood, efforts are still needed towards elucidating how cancer cells respond to antimitotic drugs owing to cytotoxicity and resistance side effects. Here, we identified the critical G2/M transcription factor Forkhead box M1 (FOXM1) as a molecular determinant of cell response to antimitotics. We found FOXM1 repression to increase death in mitosis (DiM) due to upregulation of the BCL-2 modifying factor (BMF) gene involved in anoikis, an apoptotic process induced upon cell detachment from the extracellular matrix. FOXM1 binds to a BMF intronic cis-regulatory element that interacts with both the BMF and the neighbor gene $B \cup B 1 B$ promoter regions, to oppositely regulate their expression. This mechanism ensures that cells treated with antimitotics repress BMF and avoid DiM when FOXM1 levels are high. In addition, we show that this mechanism is partly disrupted in anoikis/antimitotics-resistant tumor cells, with resistance correlating with lower BMF expression but in a FOXM1-independent manner. These findings provide a stratification biomarker for antimitotic chemotherapy response.
\end{abstract}

\section{Introduction}

Drugs interfering with spindle microtubule (MT) dynamics, MT poisons, have been effectively used as chemotherapeutic agents targeting cancer cell proliferation $^{1}$. Notwithstanding substantial research elucidating their mechanism of action, how the clinical response is achieved remains elusive. MT poisons, also known as antimitotic drugs, inhibit the metaphase-to-anaphase transition by activating the spindle assembly checkpoint due to the production of defective chromosome attachments to the spindle ${ }^{2}$. Yet, cancer cells were found to display profound intra- and inter-line variation on their

\footnotetext{
Correspondence: Elsa Logarinho (elsa.logarinho@ibmc.up.pt)

${ }^{1}$ i3S - Instituto de Investigação e Inovação em Saúde, Universidade do Porto, 4200-135 Porto, Portugal

${ }^{2}$ Aging and Aneuploidy Group, IBMC - Instituto de Biologia Molecular e Celular, Universidade do Porto, 4200-135 Porto, Portugal

Full list of author information is available at the end of the article

Edited by B. Zhivotovsky
}

response to antimitotic drugs. This variability is determined by two competing networks: one that protects Cyclin B1 from degradation and another that leads to caspase activation $^{3}$. If Cyclin B1 levels drop below the threshold needed to maintain Cdk1 activity during prolonged exposure to antimitotics, the cell exits mitosis as a polyploid entity (slippage). If apoptotic signals accumulate faster than Cyclin B1 degradation, the cell dies in mitosis (death in mitosis, DiM). Consequently, understanding the cross-talk between these two pathways is critical to foresee and modulate chemotherapy efficacy.

The Forkhead Box M1 (FOXM1) transcription factor primarily regulates the proliferation-associated gene cluster critical to $G 2 / M$ transition, mitotic spindle assembly, and chromosome segregation ${ }^{4,5}$. Aberrant FOXM1 overexpression is found in most human cancers and is a major adverse prognostic marker ${ }^{6,7}$. Moreover, FOXM1 upregulation has been implicated in the

\section{(c) The Author(s) 2021}

(c) (i) Open Access This article is licensed under a Creative Commons Attribution 4.0 International License, which permits use, sharing, adaptation, distribution and reproduction cc) in any medium or format, as long as you give appropriate credit to the original author(s) and the source, provide a link to the Creative Commons license, and indicate if changes were made. The images or other third party material in this article are included in the article's Creative Commons license, unless indicated otherwise in a credit line to the material. If material is not included in the article's Creative Commons license and your intended use is not permitted by statutory regulation or exceeds the permitted use, you will need to obtain permission directly from the copyright holder. To view a copy of this license, visit http://creativecommons.org/licenses/by/4.0/. 
development of chemotherapeutic resistance, viz. to antimitotic paclitaxel $^{8-14}$, even though the molecular mechanisms remain largely unknown.

Resistance to paclitaxel has been associated with evasion to anoikis ${ }^{15-18}$, an apoptotic process that adherent cells normally undergo upon loss of contact with the extracellular matrix or neighboring cells ${ }^{19}$. Cancer cells evade this process to survive after detachment from the primary sites and be able to metastasize ${ }^{20}$. Anoikis execution has been linked to the BH3-only pro-apoptotic proteins of the mitochondrial (intrinsic) apoptotic pathway. In particular, BMF (BCL-2-modifying factor) and BIM (BCL2L11, BCL2 like 11), which counteract the anti-apoptotic activity of BCL-2, BCL-xL, and MCL-1 $1^{19,21-23}$, or directly activate the pore-former proteins at the mitochondria ${ }^{24}$. In addition, BMF interacts with the dynein light chain DYNLL1 and DYNLL2 isoforms ${ }^{25}$, suggesting a putative role in cytoskeleton stress sensing ${ }^{26}$.

Here, we investigated whether FOXM1 expression levels determine cell fate decision upon antimitotic drug treatment. Using time-lapse live-cell imaging to monitor individual mitotic cells, we found that FOXM1 repression leads to increased DiM due to upregulation of the proapoptotic $B M F$ gene. FOXM1 binding to a $B M F$ intronic cis-regulatory element (CREs) acts to repress $B M F$ expression while enhancing the expression of the $B U B 1 B$ neighbor gene. Through this essential mechanism, mitotic cells are able to circumvent anoikis induction during prolonged arrest unless FOXM1 is repressed. However, this mechanism is partially disrupted in anoikis-resistant tumor cells. Resistance to anoikis-inducing drugs, including paclitaxel, correlates with lower levels of $B M F$ in a FOXM1-independent manner. This provides a useful biomarker for stratification of tumor response to antimitotic chemotherapy.

\section{Results}

FOXM1 expression modulates cell fate profile in response to antimitotics

We used time-lapse imaging of human dermal fibroblasts (HDFs) (young vs. aged) with distinct FOXM1 levels (high vs. low) ${ }^{27}$ to investigate individual cell response (slippage/exit vs. DiM) to antimitotic drugs (Fig. 1a). To ensure maximal mitotic blockage and apoptosis response, we treated cells with saturating concentrations of different antimitotic drugs, namely $S$-trityl-L-cysteine (STLC) that inhibits kinesin-5/Eg5, and the classic MT poison paclitaxel (TX). In both, 87-year-old HDFs (low FOXM1) were lentiviral-transduced to express a constitutively active form of FOXM1 (FOXM1 OX) (Fig. 1b; Fig. S1a), whereas 10-year-old HDFs (high FOXM1) were transfected with FOXM1 siRNA (siFOXM1) (Fig. 1c; Fig. S1b). We found that FOXM1 induction in elderly cells shifted their cell fate profile towards mitotic exit in both STLC (Fig. 1d, e) and
TX treatments (Fig. S1c, d). Conversely, siRNA-mediated FOXM1 repression in young fibroblasts shifted their cell fate profile towards DiM in comparison with controls (Fig. 1g, h; Fig. S1f, g), which is in agreement with increased DiM also found in aged vs. young HDFs. The siRNAdriven shift towards DiM was not due to any differences in antimitotic drug action (Fig. S1i), and was specifically due to FOXM1 repression, as evaluated by FOXM1 OX rescue experiment (Fig. S1j, k). We additionally measured the time cells took to execute exit or DiM in response to STLC and TX. We found FOXM1 induction to accelerate slippage and FOXM1 repression to accelerate DiM (Fig. 1f, i; Fig. S1e, h), thus suggesting that FOXM1 modulates both competing networks. Moreover, we assessed the degradation rate of GFP-tagged Cyclin B1 in young and elderly cells (Fig. S2a, b). Despite the lower Cyclin B1 levels in elderly cells as previously reported ${ }^{27}$, we observed that cyclin B1 is degraded slower in elderly cells (Fig. S2b) thereby explaining why these cells are not more slippageprone than younger cells. This is possibly owing to downregulation of the Cdc20 co-factor of APC/C activity $^{27}$. In addition, we tested non-RNAi modalities for FOXM1 repression, namely the small-molecule inhibitors RCM-1 ${ }^{28}$, which inhibits FOXM1 nuclear localization, and FDI- $6^{29}$, which inhibits FOXM1 binding to DNA. Both inhibitors shifted cell fate profiling of young cells towards increased DiM in response to paclitaxel (Fig. S2c-h), supporting that FOXM1 repression promotes DiM via its canonical role as transcription factor.

\section{BMF is an effector of DiM driven by FOXM1 inhibition in response to antimitotics}

As FOXM1 inhibition accelerates DiM, this suggests that FOXM1 influences the death pathway of the competing networks model. Thus, we interrogated the "regulation of cell death" GO term in our previous RNA-seq data sets generated from mitotic HDFs blocked with STLC ${ }^{27}$ (Fig. 2a). We found 64 differently altered genes across the neonatal vs. 87 y HDFs, mock vs. siFOXM1 10 y HDFs, and empty vs. FOXM1 OX 87 y HDFs RNA-seq data sets. Fortyeight out of these 64 genes exhibited similar transcriptional changes in elderly and siFOXM1-depleted cells, which were reverted upon FOXM1 overexpression (Fig. 2b). The most downregulated gene in FOXM1 RNAi was BIRC5, which encodes the well-established pro-survival factor known as Survivin. The most upregulated gene was $B M F$, which similarly to $B B C 3$ (PUMA) and PMAIP1 (NOXA), encodes for a BH3-only pro-apoptotic protein ${ }^{30}$. BMF captured our attention because, like BIM, it is reported as an effector of anoikis ${ }^{19}$, and anoikis has been associated with resistance to paclitaxel $^{15-18}$. Thus, we wondered whether FOXM1 repression in cells under prolonged mitotic arrest shifts their fate towards DiM through $B M F$ upregulation and anoikis induction. qPCR analysis confirmed $B M F$ 


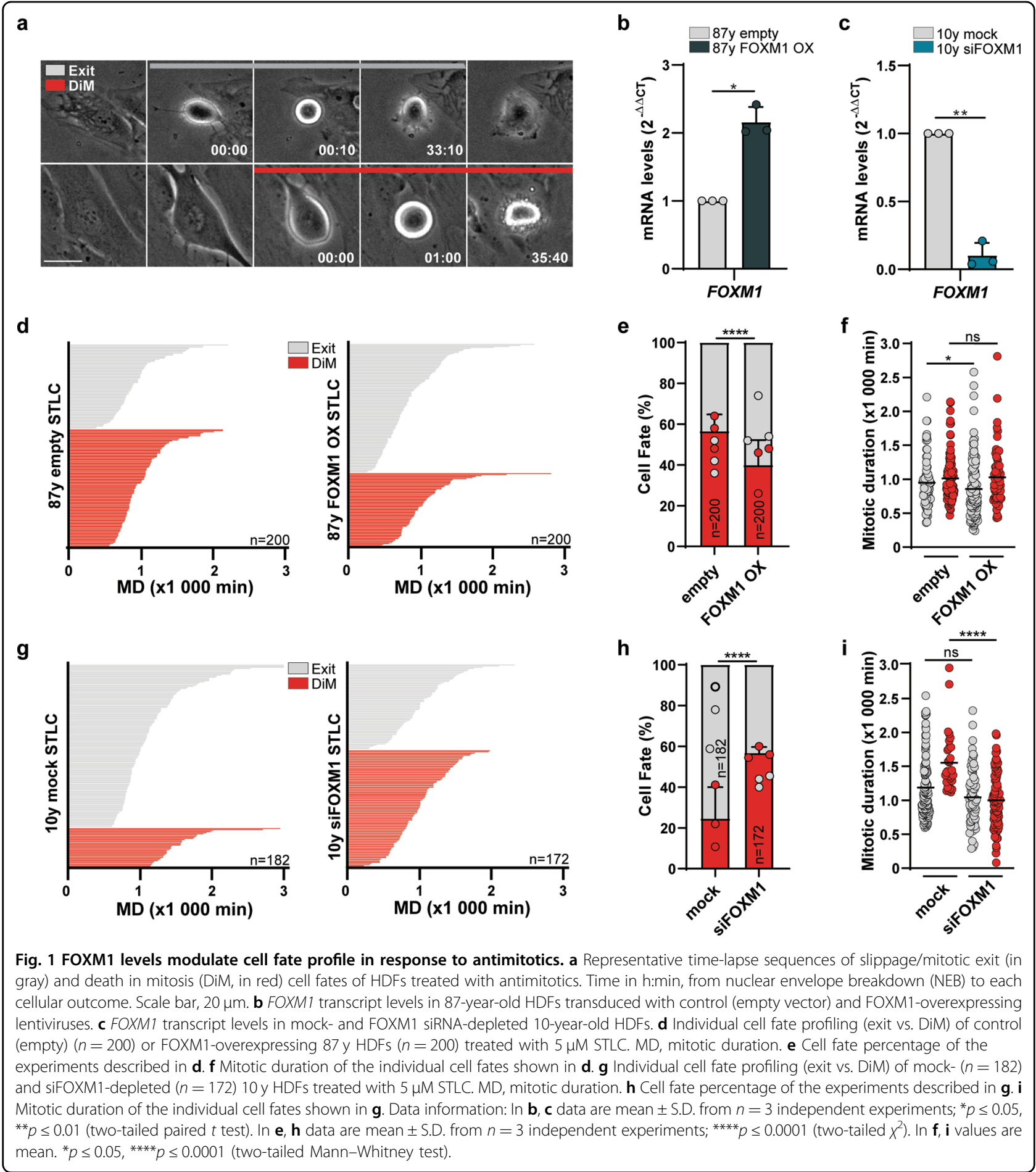

upregulation in elderly (87 y) and siFOXM1-depleted young $(10 \mathrm{y})$ cells arrested in mitosis with STLC in comparison with controls (Fig. 2c-d). BMF protein levels could not be monitored owing to lack of available specific antibodies as reported by others ${ }^{19,31}$. To address the functional role of BMF in mitotic cell fate response to antimitotics, neonatal
HDFs (HDF N) were lentiviral-transduced to express FLAG-tagged BMF at an efficiency of $\sim 40 \%$ (Fig. S3a, b). We found that BMF overexpression significantly increased and accelerated DiM in response to STLC (Fig. 2e-g) and TX (Fig. S3c-e). Conversely, CRISPR/Cas9-mediated knockout of $B M F$ (Fig. S3f, g) significantly rescued the shift 


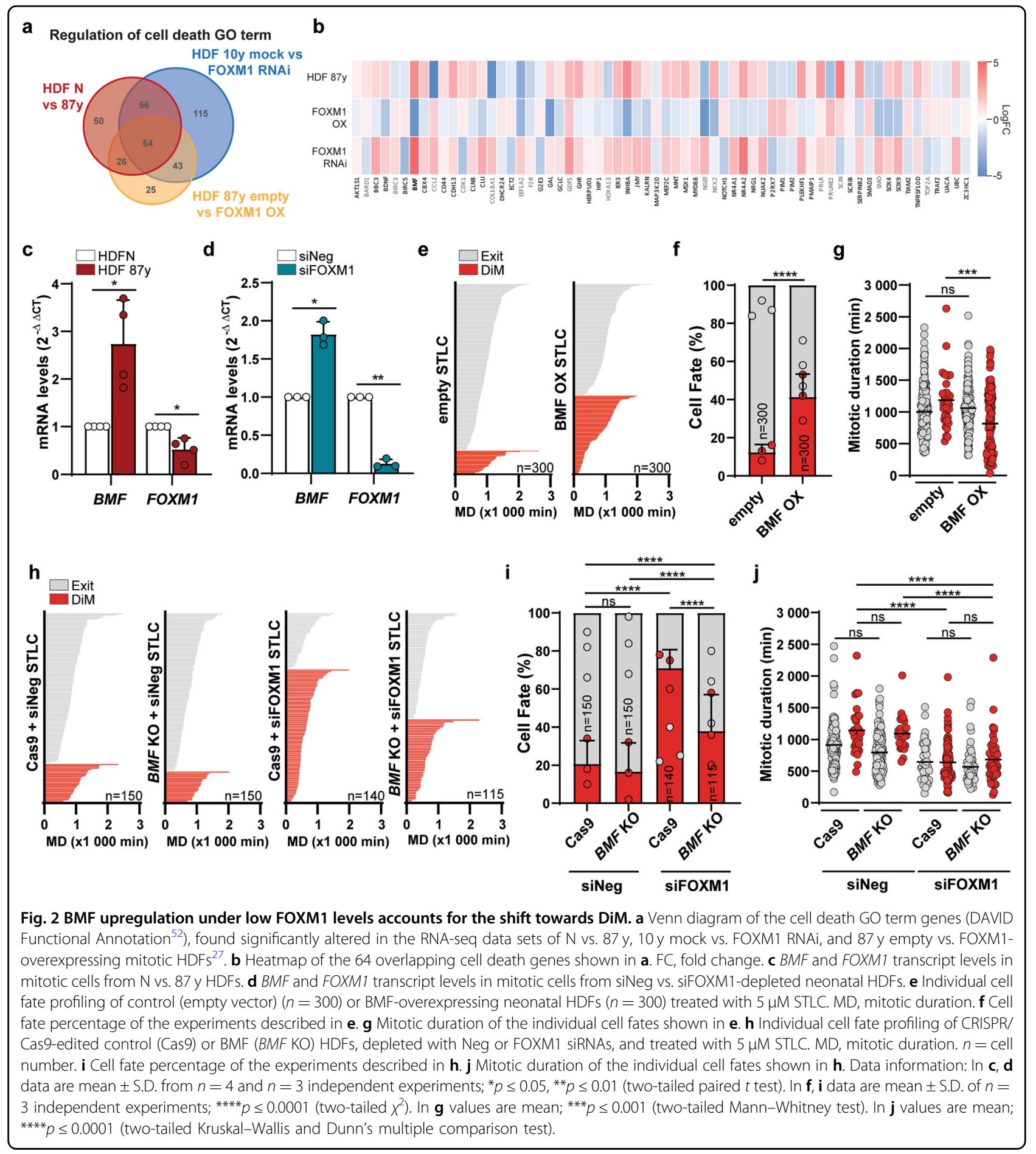

towards DiM observed in siFOXM1- vs. siNeg-depleted cell cultures treated with STLC and TX (Fig. 2h-i; Fig. S3h-i). However, $B M F$ knockout did not alter the time cells took to exit or die in mitosis, suggesting that the cyclin B1 degradation pathway remained unchanged (Fig. 2j; Fig. S3j). In addition, we verified that in the absence of antimitotics, neither FOXM1 repression nor BMF overexpression was able to induce DiM (Fig. S4a-c), thus indicating that a mitotic delay is needed to mount the apoptotic signaling, and that FOXM1 and BMF modulate cell death under priming conditions of prolonged mitotic arrest. Overall, these data show that BMF accounts for increased DiM in response to antimitotic drugs under low FOXM1 transcriptional activity. 


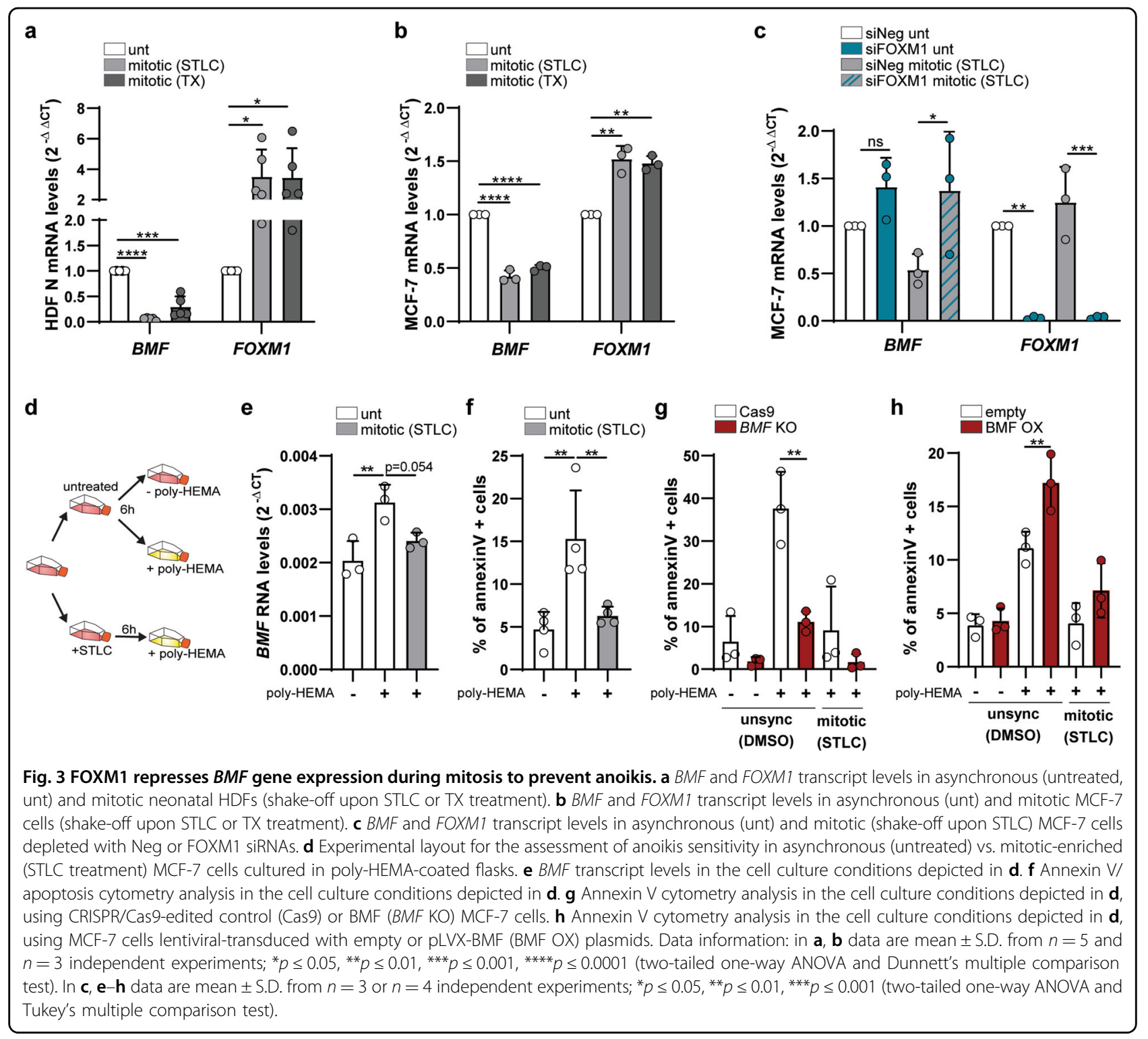

\section{FOXM1 is required to inhibit $B M F$ expression during mitosis}

As FOXM1 is active at the G2/M cell cycle transition and we found FOXM1 repression to induce $B M F$ upregulation, we hypothesized that $B M F$ gene expression is cell cycle-regulated. To test this, we performed a mitotic shake-off of neonatal cultures treated with STLC and TX. qPCR analysis of mitotic cell fractions vs. asynchronous cell population revealed pronounced downregulation of $B M F$ in arrested mitotic cells expressing high FOXM1 levels (Fig. 3a). Similar results were found using an alternative cellular model, the MCF-7 breast cancer epithelial cell line (Fig. 3b), in which FOXM1 repression also induces $B M F$ upregulation (Fig. 3c). In addition, qPCR analysis of an MCF-7 G2-enriched cell population retrieved $7 \mathrm{~h}$ upon thymidine block washout (before apparent mitotic roundup) (Fig. S5a) confirmed that $B M F$ is already downregulated in G2 and thus, not merely due to the global transcriptional shutdown during mitosis (Fig. $\mathrm{S} 5 \mathrm{~b})$.

Next, we asked whether $B M F$ repression during G2/M is critical to prevent anoikis during prolonged mitotic arrest. To test this idea, we plated asynchronous and mitosis-enriched ( $6 \mathrm{~h}$ STLC treatment) MCF-7 cell populations in poly-2-hydroxyethyl methacrylate (polyHEMA)-coated dishes to measure the extent of anoikis induced by the poly-HEMA inhibition of cell adherence (Fig. 3d). qPCR analysis of BMF expression (Fig. 3e) and annexin $\mathrm{V}$ cytometry (Fig. 3f) were used as readouts for anoikis after $6 \mathrm{~h}$ cell culture in poly-HEMA-coated dishes. We found $B M F$ upregulation and increased apoptosis in the MCF-7 asynchronous cell population (mostly G1, see 
Fig. S5a) but not in the mitotic subpopulation. Apoptosis in the MCF-7 asynchronous population was specifically due to BMF, as evaluated by a rescue experiment using $B M F$ KO MCF-7 cells (Fig. 3g). Conversely, BMF overexpression in MCF-7 cells turned cells more sensitive to poly-HEMA (Fig. 3h). Thus, we conclude that BMF expression is cell cycle-regulated, being repressed during G2/M when FOXM1 is transcriptionally active. Importantly, this regulation precludes prolonged mitotic cell rounding from being perceived as cell detachment and from inducing anoikis.

\section{FOXM1 acts as a $B M F$ transcriptional repressor}

To gain insight into the mechanism by which FOXM1 represses $B M F$ transcription during mitosis, we searched for potential FOXM1-binding sites in the $B M F$ genomic region. Interrogation of a publicly available FOXM1 ChIP-seq data set for MCF-7 cells ${ }^{32}$ revealed the BMF promoter, as well as several intronic regions with an $\mathrm{H} 3 \mathrm{~K} 27 \mathrm{ac}$ mark for active $\mathrm{CREs}^{33}$, as FOXM1-binding sites (Fig. 4a). To assess the role of FOXM1 binding at the $B M F$ promoter, we performed a promoter-luciferase reporter assay in MCF-7 siNeg- and siFOXM1-depleted cells. We found $B M F$ promoter activity in comparison with negative control in siNeg-depleted cells, further enhanced upon siRNA-mediated depletion of FOXM1 (Fig. 4b). This FOXM1 transcriptional repression at the $B M F$ promoter is not through promoter methylation as previously reported for GATA3, in which FOXM1 was shown to recruit the DNA methylase DNMT3B ${ }^{34}$ (Fig. S6a). Also, we excluded transcriptional activation by FOXO3 as a competing mechanism. Although $B M F$ has been described as FOXO3 transcriptional target ${ }^{21}$, and FOXO3 and FOXM1 to function antagonistically in cancer cells ${ }^{35}$, we found FOXO3 depletion to induce $B M F$ upregulation and FOXM1 downregulation in HDFs (Fig. S6b).

As CREs can modulate gene expression, we deleted the putative CREs\#1-3 (Fig. 4a) using CRISPR/Cas9-mediated genomic editing. CRE\# 1 and CRE\#2 deletions did not change $B M F$ gene expression in polyclonal MCF-7 cell populations (Fig. 4c, d; Fig. S6c, d). Regarding CRE\#3 deletion, we repeatedly failed to establish polyclonal cultures from CRE\#3-deleted cells (Fig. S6e). This was not due to single guide RNAs (sgRNAs) inefficacy, as successful genomic deletion was detected immediately after fluorescence-activated cell sorting (FACS) (Fig. S6f). Importantly, qPCR analysis in these FACS-sorted CRE\#3deleted cells revealed $B M F$ upregulation (Fig. 4e), thus establishing this FOXM1-binding CRE as a transcriptional $B M F$ silencer. In addition, we confirmed that FOXM1 RNAi, CRE\#3 genomic deletion, and their combination, all exhibit equivalent effect in $B M F$ upregulation, which supports that FOXM1 represses $B M F$ through its binding to CRE\#3 (Fig. 4f). To confirm that cell death driven by $B M F$ upregulation, and not $B U B 1 B$ downregulation, was precluding the establishment of a polyclonal cell population deleted for CRE\#3, we performed the CRISPR/Cas9mediated CRE\#3 deletion in BMF KO (Fig. 4g) and in $B U B 1 B$ OX (Fig. S6g-i) MCF-7 cells. Under the BMF KO genetic background, but not under $B U B 1 B O X$, we were able to establish a polyclonal cell population deleted for CRE\#3 (Fig. 4g), which demonstrates that CRE\#3 is required for cell viability. Overall, our data disclosed the FOXM1-binding CRE\#3 as a transcriptional $B M F$ silencer.

\section{CRE within $B M F$ intronic region works bifunctionally as $B U B 1 B$ enhancer and $B M F$ silencer to inhibit anoikis during prolonged mitosis}

To gain further insight into the functional role of CRE\#3, and considering the presence of the H3K27ac mark from the available ChIP-seq data set ${ }^{33}$, we performed an enhancer-luciferase reporter assay. We found equivalent enhancer activity in cells transfected with the CRE\#3-containing reporter plasmid in comparison with the positive control (Tyrosine kinase promoter) (Fig. 4h). Moreover, FOXM1 repression reduced the CRE\#3 regulatory activity. Interestingly, we noticed that in the vicinity of the $B M F$ genomic region is the $B U B 1 B$ spindle assembly checkpoint gene, an established FOXM1 transcriptional mitotic target. qPCR analysis of CRISPR/Cas9 CRE\#3-deleted cells immediately after FACS revealed decreased $B U B 1 B$ transcript levels (Fig. 4i), supporting that CRE\#3 is a $B U B 1 B$ enhancer. It is known that coregulated genes are normally confined in close proximity and that CREs and promoters are brought together by chromatin looping ${ }^{36}$. To ascertain chromatin interactions between CRE\#3 and the BMF and BUBR1 promoter regions, we performed $4 \mathrm{C}$-sequencing analysis with the viewpoint on CRE\#3. We found that $B M F$ promoter, $B U B 1 B$ promoter, and CRE\#3 physically interact (Fig. 4j). Thus, our data disclose an unforeseen FOXM1-binding CRE that acts to induce $B U B 1 B$ transcription while repressing $B M F$ expression. We propose that through this mechanism, mitotic cells are able to circumvent cell death signaling induced by prolonged mitotic arrest.

\section{High $B M F$ levels correlate with response to anoikis- inducing chemotherapeutic drugs}

To extend our findings into antimitotic chemotherapy response, we investigated the FOXM1-dependent co-regulation of $B M F$ and $B U B 1 B$ expression in the context of tumor cell treatment. As a preliminary approach, we used the MCF-7 and MDA-MB-231 breast cancer cell lines reported as anoikis-sensitive and anoikis-resistant, respectively $^{21}$. In agreement, we confirmed higher expression of $B M F$ in MCF-7 vs. MDA-MB-231 cells, even though 


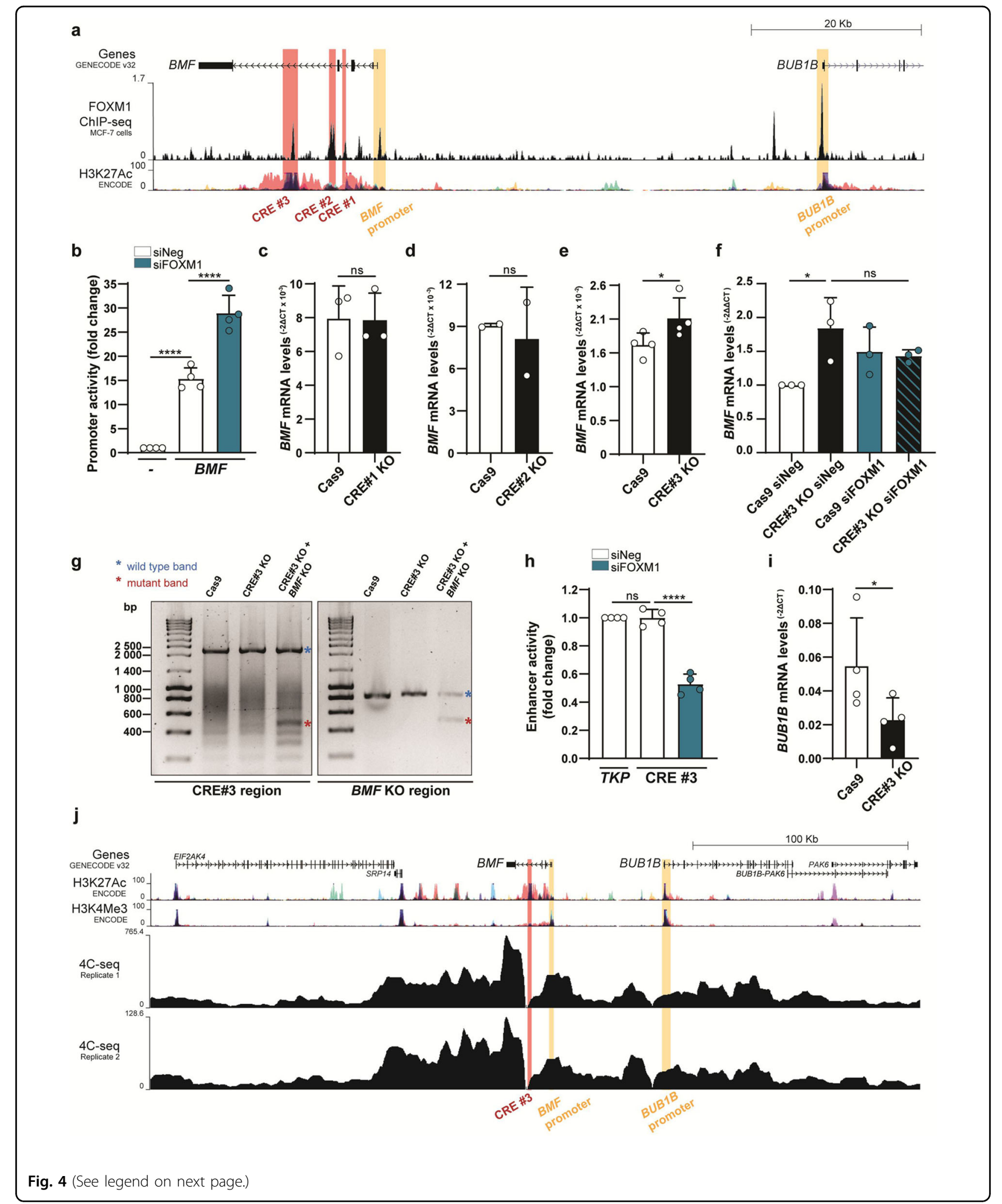

FOXM1 and $B U B 1 B$ transcript levels were similar in both cell lines (Fig. 5a). We then evaluated if $B M F$ expression is cell cycle-regulated in a FOXM1-dependent manner in
MDA-MB-231 cells, as we did for the MCF-7 cells (see Fig. 3c). Interestingly, qPCR analysis of mitotic vs. asynchronous MDA-MB-231 cell populations revealed no significant 
(see figure on previous page)

Fig. 4 FOXM1-binding CRE within $B M F$ intronic region represses $B M F$ while enhancing $B U B 1 B$ expression. a $B M F$ and $B U B 1 B$ genomic landscape from UCSC genome browser with tracks of FOXM1 ${ }^{32}$ and H3K27AC ${ }^{33}$ ChIP-seq data. b BMF promoter-reporter assay in siNeg- and siFOXM1-depleted MCF-7 cells. c BMF transcript levels in CRE\#1 CRISPR/Cas9-deleted MCF-7 polyclonal cell population. d BMF transcript levels in CRE\#2 CRISPR/Cas9-deleted MCF-7 polyclonal cell population. e BMF transcript levels in CRE\#3 CRISPR/Cas9-deleted MCF-7 cells immediately after sorting. f BMF transcript levels in CRE\#3 CRISPR/Cas9-deleted and siFOXM1-depleted MCF-7 cells immediately after sorting. $\mathbf{g}$ Genomic PCR validation for the ability to generate CRE\#3 CRISPR/Cas9-deleted polyclonal cell population only in a BMF KO background. $\mathbf{h}$ Enhancer reporter assay for CRE\#3 in siNeg- and siFOXM1-depleted MCF-7 cells. i BUB1B transcript levels in CRE\#3 CRISPR/Cas9-deleted MCF-7 cells immediately after sorting. $\mathbf{j} 4$ 4-seq replicates of neonatal HDFs with a viewpoint in CRE\#3 (red). BMF promoter and BUB1B promoter interactions with CRE\#3 are highlighted (orange). Data information: in $\mathbf{b}, \mathbf{f}, \mathbf{h}$ data are mean \pm S.D. from $n=3$ or $n=4$ independent experiments; ${ }^{* * * *} p \leq 0.0001$ (two-tailed one-way ANOVA and Tukey's multiple comparison test). In $\mathbf{c}$, $\mathbf{d}$ data are mean \pm S.D. from $n=3$ and $n=2$ independent experiments; $n s, p \leq 0.05$ (two-tailed

Mann-Whitney test). In $\mathbf{e}$, i data are mean \pm S.D. from $n=4$ independent experiments; ${ }^{*} p \leq 0.05$ (two-tailed paired $t$ test).

changes in $B M F$ expression, even upon FOXM1 RNAi (Fig. 5b). Accordingly, time-lapse imaging of siNeg- and siFOXM1-depleted MCF-7 and MDA-MB-231 cells treated with paclitaxel confirmed a higher resistance of MDA-MB231 cells in comparison with MCF-7 cells as described ${ }^{37}$, and revealed that FOXM1 repression only modestly increases DiM in the MDA-MB-231 anoikis-resistant cells in comparison with the MCF-7 cells (Fig. 5c-f). Similar results were obtained when using small-molecule inhibition of FOXM1 with FDI-6 (Fig. S7a-d). Conversely, we also confirmed FOXM1 overexpression in MDA-MB-231 cells not to change BMF expression (Fig. S7e, f). Thus, these data suggest that anoikis-resistant cells are able to repress $B M F$ in a FOXM1-independent manner. Still, CRISPR/Cas9-mediated CRE\#3 deletion impairs MDA-MB-231 cell viability and induces $B M F$ upregulation (Fig. S7g-i), suggesting that CRE\#3 is required for $B M F$ repression, although not through FOXM1 as in MCF-7 cells.

To examine $B M F$ expression in a wider tumor context, we interrogated the Cancer Cell Line Encyclopedia $(C C L E)^{38}$ for the association between mRNA and protein expression levels with drug responses in the Cancer Target Discovery and Development $\left(\mathrm{CTD}^{2}\right)$ database ${ }^{39}$, specifically for chemotherapeutics most extensively reported as anoikis-inducing: paclitaxel, docetaxel, and doxorubicin. We found a significant correlation of higher $B M F$ mRNA and protein levels with response to these drugs (Fig. $5 \mathrm{~g}$, h; Table S1). Interestingly, higher BUB1B mRNA and protein levels also correlated with increased drug sensitivity as observed for $B M F$, suggesting the disruption of the FOXM1-dependent co-regulatory mechanism found in untransformed and MCF-7 cells (Fig. 5j, k; Table S1). Accordingly, FOXM1 mRNA and protein levels did not show a correlation with any drug (Table S1). Thus, this analysis supports that tumor cells with lower $B M F$ expression are more resistant to anoikis-inducing drugs, and independently of FOXM1 expression.

To further examine the clinical relevance of these findings, we interrogated microarray data sets from XeNA, a clinical trial examining response rates in women with operable, early-stage breast cancer receiving neoadjuvant capecitabine plus the antimitotic agent docetaxel ${ }^{40}$. Tumors from patients showing no response had diminished $B M F$ expression (Fig. 5i). Consistent with the premise that $B M F$ expression in these non-responsive patients is FOXM1-independent, we found no significant elevated expression in the case of $B U B 1 B$ (Fig. 5l).

\section{Discussion}

Our study brings insight into the poorly understood mechanisms that define the kinetics of death signaling accumulation and threshold during mitotic arrest induced by antimitotic chemotherapy. We show that FOXM1 transcriptional activity driving G2/M cell cycle gene expression, also modulates the apoptotic pathway of the competing networks model. Both genetic and pharmacological repressions of FOXM1 increased DiM upon antimitotic drug treatment. Previous studies have reported a correlation between FOXM1 repression and paclitaxel response, with targets including genes involved in MT dynamics or drug efflux ${ }^{8-12}$. Here, we originally identified an apoptotic gene repressed by G2/M FOXM1 transcriptional activity, $B M F$. FOXM1 binds to the $B M F$ promoter and an intronic CRE\#3 to inhibit its expression. Interestingly, we found that transcription of the $B M F$ neighbor gene, $B U B 1 B$, which is a FOXM1 transcriptional target, is coupled with $B M F$ repression. We showed that the $B M F$ promoter, $B U B 1 B$ promoter, and CRE\#3 physically interact, with the FOXM1-binding CRE operating bifunctionally to induce $B U B 1 B$ transcription, whereas silencing $B M F$ (Fig. 6). Curiously, this resembles an antithetical regulation of endothelial $A C E$ and $A C E 2$ by a BRG1-FOXM1 complex previously reported in the context of pathologic cardiac hypertrophy ${ }^{41}$.

Importantly, the transcriptional regulation here described explains how cells with low FOXM1 levels become more prone to DiM upon antimitotic drug treatment. $B M F$ genomic deletion was able to revert increased DiM under low FOXM1 transcriptional activity, disclosing anoikis as an unforeseen apoptotic program in response 


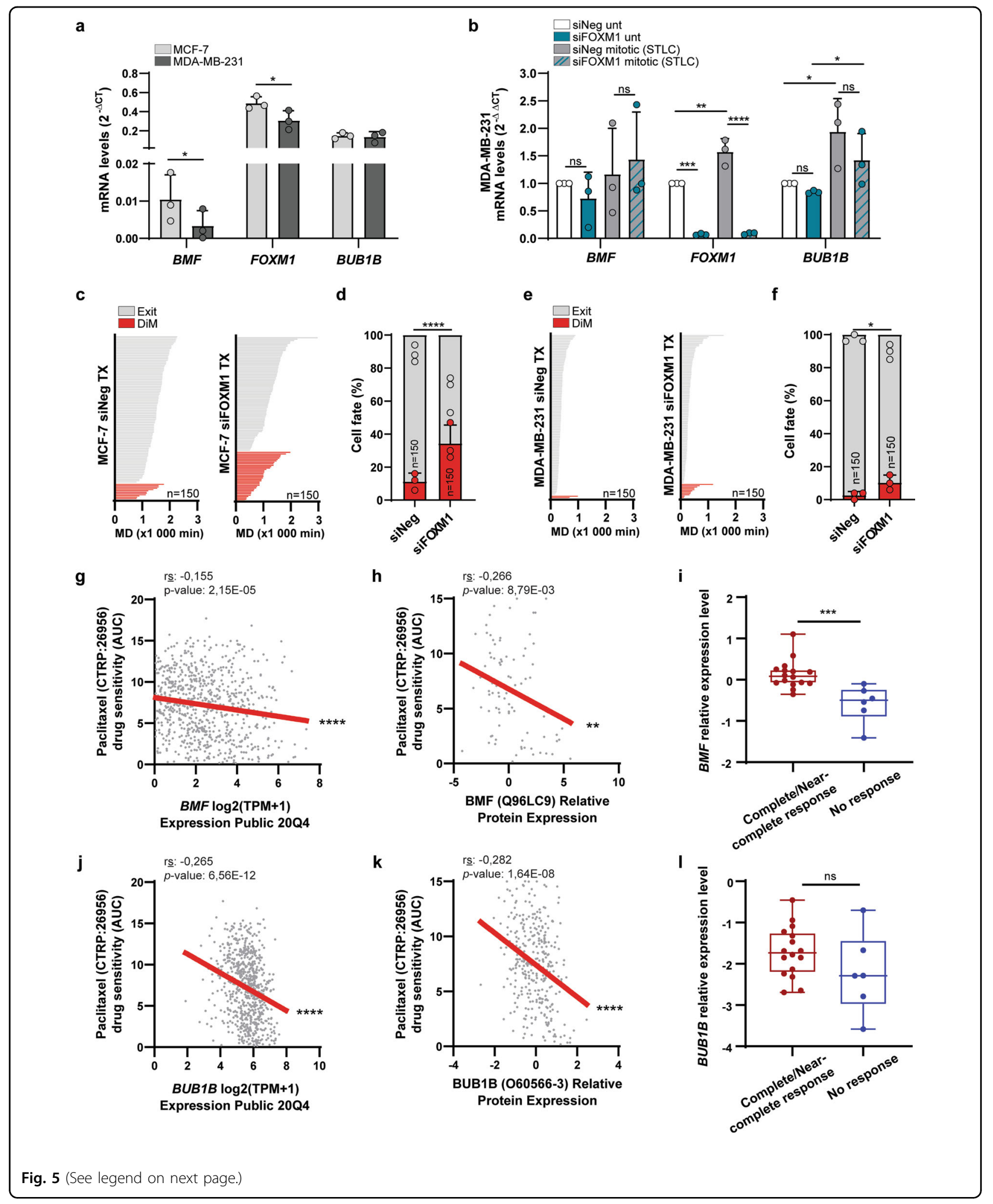

to antimitotics. This supports the idea of using FOXM1 inhibition to improve antimitotic drug response. However, when extrapolating our findings into a wide tumor context, we found that FOXM1-dependent BMF repression appears disrupted in anoikis-resistant cells. Interrogation of the CCLE for the association between FOXM1 
(see figure on previous page)

Fig. 5 BMF expression correlates with response to antimitotic drug chemotherapy. a $B M F, F O X M 1$, and $B U B 1 B$ transcript levels in MCF-7 and MDA-MB-231 cells. b BMF, FOXM1, and BUB1B transcript levels in asynchronous (untreated, unt) and mitotic (shake-off upon STLC) MDA-MB-231 cells depleted with Neg or FOXM1 siRNAs. c Individual cell fate profiling of siNeg- $(n=150)$ or siFOXM1-depleted $(n=150)$ MCF-7 cells treated with 500 nM TX. MD mitotic duration. d Cell fate percentage of the experiments described in c. e Individual cell fate profiling of siNeg- $(n=150)$ or siFOXM1-depleted ( $n=150$ ) MDA-MB-231 cells treated with $500 \mathrm{nM} \mathrm{TX.} \mathrm{MD,} \mathrm{mitotic} \mathrm{duration.} \mathbf{f}$ Cell fate percentage of the experiments described in e. $\mathbf{g}$ Correlation of BMF mRNA levels and paclitaxel drug sensitivity in the CTRP drug response data set. $\mathbf{h}$ Correlation of BMF protein levels and paclitaxel drug sensitivity in the CTRP drug response data set. i Relative BMF mRNA expression levels in tumors with complete/near-complete response vs. no response to a combination of docetaxel and capecitabine ${ }^{40}$. $\mathbf{j}$ Correlation of $B \cup B 1 B$ mRNA levels and paclitaxel drug sensitivity in the CTRP drug response data set. $\mathbf{k}$ Correlation of BUB1B protein levels and paclitaxel drug sensitivity in the CTRP drug response data set. I Relative BUB1B mRNA expression levels in tumors with complete/near-complete response vs. no response to a combination of docetaxel and capecitabine ${ }^{40}$. Data information: In a data are mean \pm S.D. from $n=3$ independent experiments; ${ }^{*} p \leq 0.05$ (two-tailed paired $t$ test). In $\mathbf{b}$ data are mean $\pm S$.D. from $n=3$ independent experiments; ${ }^{*} p \leq 0.05,{ }^{* *} p \leq 0.01,{ }^{* * *} p \leq 0.001,{ }^{* * * *} p \leq 0.0001$ (two-tailed one-way ANOVA and Tukey's multiple comparison test). In $\mathbf{d}, \mathbf{f}$ data are mean \pm S.D. of $n=3$ independent experiments; ${ }^{*} p \leq 0.05$, ${ }^{* * *} p \leq 0.0001$ (two-tailed $x^{2}$ ). In $\mathbf{g}, \mathbf{h}, \mathbf{j}, \mathbf{k}{ }^{* *} p \leq 0.01$, ${ }^{* * *} p \leq 0.0001$ (https:// depmap.org/portal/interactive/). In $\mathbf{i}, \mathbf{I}{ }^{* * *} p \leq 0.001$, ns $p>0.05$ (two-tailed Mann-Whitney test).

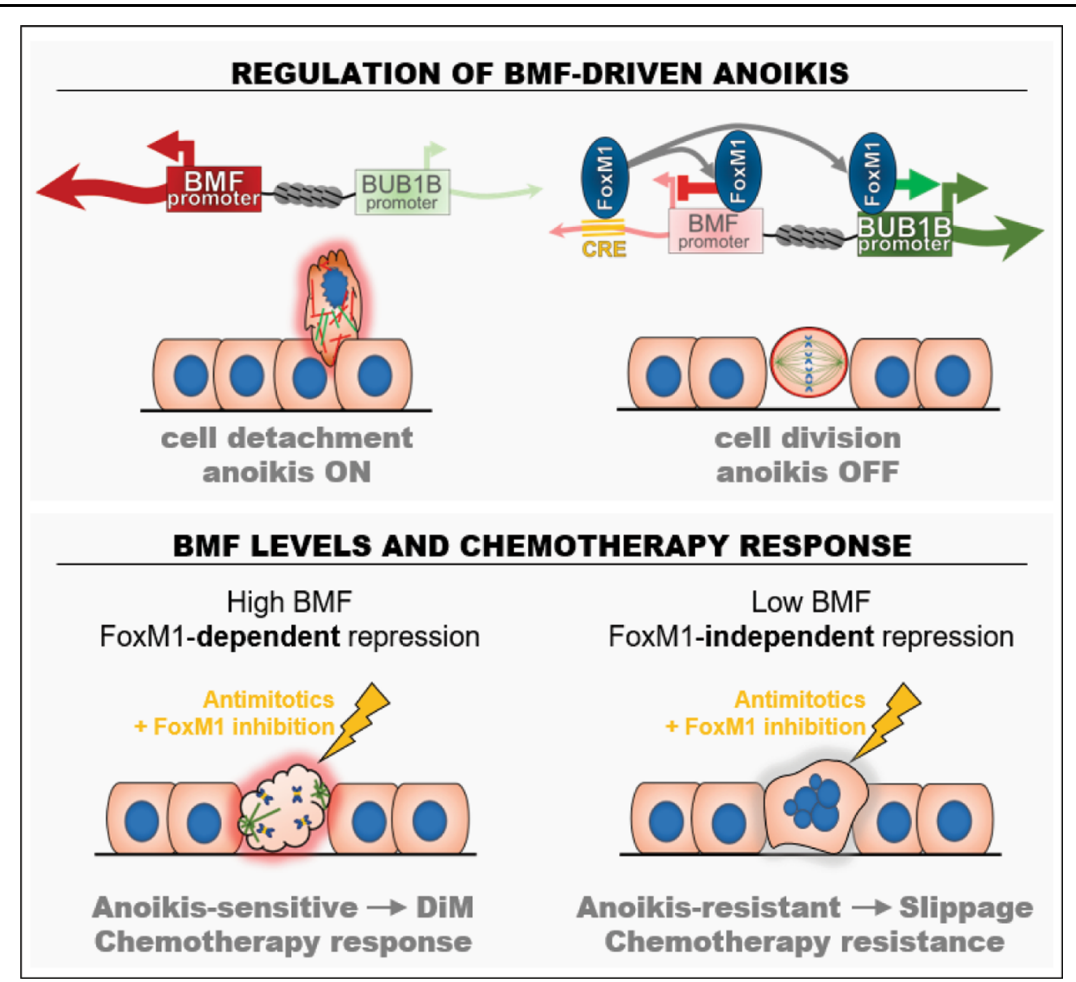

Fig. 6 Working model integrating BMF transcriptional regulation by FOXM1 in mitosis and antimitotic chemotherapy. Upper panel: FOXM1 inhibits BMF expression and anoikis induction during prolonged mitosis. A FOXM1-binding cis-regulatory element (CRE) in BMF is an enhancer of the $B U B 1 B$ neighbor mitotic gene. Lower panel: FOXM1 inhibition increases DiM in response to antimitotic drugs in anoikis-sensitive cells (high BMF). FOXM1-independent BMF repression (low BMF) correlates with resistance to antimitotic chemotherapy.

and $B M F$ expression levels with anoikis-inducing drug responses in the $\mathrm{CTD}^{2}$ database revealed a significant correlation only for $B M F$. In agreement, we found FOXM1-independent $B M F$ repression in the anoikisresistant MDA-MB-231 cell line, whereas FOXM1 inhibition still upregulated $B M F$ and increased DiM in the anoikis-sensitive MCF-7 cell line. Although the detailed mechanism of FOXM1-independent BMF repression remains unclear, we found that CRE\#3 is still essential. Importantly, our findings disclose $B M F$ levels as predictive of tumor response to antimitotic chemotherapy, with only higher levels supporting combination therapy of FOXM1 inhibitors with antimitotics (Fig. 6).

Other mechanisms have been reported to influence the timing of mitotic cell death, such as degradation of Mcl$1^{42}$, Cdk1-dependent caspase phosphorylation ${ }^{43}$, c-Myc 
overexpression $^{44}$, and cGAS activation ${ }^{45}$. Whether these mechanisms cross-talk with the FOXM1 transcriptional competence and BMF apoptotic potential remains unknown. FOXM1 has been involved in regulatory loops with $\mathrm{Cdk} 1^{46,47}$ and $\mathrm{c}-\mathrm{Myc}^{48,49}$, with $\mathrm{Cdk} 1$ and $\mathrm{c}-\mathrm{Myc}$ upregulating FOXM1 activity, as well as the other way round. One interesting aspect about FOXM1 is that it regulates targets of the two competing networks determining fate decision upon mitotic arrest, with this study disclosing its role in the apoptotic pathway. Further investigation on how FOXM1 transcriptional activity becomes uncoupled from BMF-driven apoptosis will contribute to our understanding of chemotherapy response.

\section{Methods and materials Cell culture}

HDFs from healthy Caucasian males were obtained from the Coriell Cell Repository (NJ, USA) (HDF 10 y GM03348, HDF 87 y AG10884) and Zen Bio (NC, USA) (HDF N DFM021711A) and kept at a low passage number. HDFs were cultured in minimal essential medium Eagle-Earle (MEM) supplemented with 15\% fetal bovine serum (FBS), $2.5 \mathrm{mM}$ L-glutamine, and $1 \times$ antibiotic-antimycotic (all from Gibco, Thermo Fisher Scientific, CA, USA). MCF-7 cells (ATCC HTB-22) and MDA-MB-231 (HTB-26) were obtained from ATCC (VA, USA) and cultured with Dulbecco's modified Eagle's medium GlutaMAX (DMEM) (Gibco, Thermo Fisher Scientific, CA, USA) supplemented with $10 \% \mathrm{FBS}$ and $1 \times$ antibiotic-antimycotic. All cells were grown in ventilated flasks at $37{ }^{\circ} \mathrm{C}$ and humidified atmosphere with $5 \% \mathrm{CO}_{2}$.

\section{Drug treatments}

$S$-Trityl-L-cysteine (STLC, Tocris, UK) was used at $5 \mu \mathrm{M}$, and paclitaxel (TX, Sigma-Aldrich, MO, USA) at $500 \mathrm{nM}$. For mitotic shake-off, cells were treated with antimitotic drugs for $16 \mathrm{~h}$. For live-cell imaging, cells were treated for 24 $\mathrm{h}$ with FDI-6 (Axon Medchem, VA, USA) and RCM-1 (kindly provided by Vladimir Kalinichenko, $\mathrm{OH}$, USA). In all, $1 \mathrm{mM}$ thymidine block for $20 \mathrm{~h}$ followed by a $7 \mathrm{~h}$ washout into the fresh medium was used for $\mathrm{G} 2$ cell synchronization.

\section{RNA interference}

HDFs were transfected with $45 \mathrm{nM}$ siRNA Universal Negative Control \#1 (Sigma-Aldrich, MO, USA), $45 \mathrm{nM}$ FOXM1 small interfering RNA (SASI_Hs01_00243977, Sigma-Aldrich, MO, USA), or $45 \mathrm{nM}$ FOXO3 small interfering RNA (SASI_HS01_00119127, Sigma-Aldrich, MO, USA) using Lipofectamine RNAiMAX (Thermo Fisher Scientific, CA, USA) according to manufacturer's instructions. MCF-7 and MDA-MB-231 were transfected with $20 \mathrm{nM}$ siRNA. Cells were analyzed $72 \mathrm{~h}$ post transfection, and depletion efficiency was validated by qPCR.

\section{Plasmid transfection}

HDFs were transfected with $500 \mathrm{ng}$ of pcDNA3/Cyclin B1-GFP (\#39871, Addgene) using Lipofectamine 3000 (Life Technologies, Thermo Scientific, CA, USA) according to the manufacturer's instructions. Cells were analyzed $48 \mathrm{~h}$ post transfection.

\section{Lentiviral production and infection}

pLVX-FOXM1-dNdK was generated as previously described in ref. ${ }^{27}$. pBabe HA human BMF plasmid (\#17239, Addgene) was digested with BamHI and EcoRI and the coding sequence inserted into the pLVX-Tight-Puro Vector (PT3996-5, Clontech, Takara Bio USA) to generate the pLVX-BMF-Flag construct. $B U B 1 B$ coding sequence was reverse-transcribed from HDF N total RNA and inserted into pLVX-Tight-Puro. Lentiviruses were produced in HEK293T cells transfected with pLVX-empty, pLVX-BMFFlag, pLVX-BUB1B, pLVX-FOXM1-dNdK or pLVX-TetOn Advanced (PT3990-5, Clontech, Takara Bio USA) and the packaging plasmids (pMd2.G and psPAX2, \#12259 and \#12260, respectively, Addgene) using Lipofectamine 2000 (Life Technologies, Thermo Scientific, CA, USA) according to manufacturers' instructions. Cells were transduced with inducible and transactivator viruses at 2:1 ratio in media containing $8 \mu \mathrm{g} / \mathrm{ml}$ polybrene (Sigma-Aldrich, MO, USA). $500 \mathrm{ng} / \mathrm{ml}$ doxycycline (Sigma-Aldrich, MO, USA) was added to fresh media and phenotypes analyzed $24 \mathrm{~h}$ later for BMF OX and $72 \mathrm{~h}$ later for FOXM1 OX. Transduction efficiency was measured by Flag immunostaining or by FOXM1/BUB1B qPCR. $1 \mu \mathrm{g} / \mathrm{mL}$ puromycin and $400 \mu \mathrm{g} / \mathrm{mL}$ G418 antibiotic selection was used to enrich MCF-7 BUB1B OX, MCF-7 BMF OX and MDA-MB-231 FOXM1 OX cells.

\section{CRISPR/Cas9-mediated deletions and validation}

sgRNAs upstream and downstream of the target region were designed using the Benchling online platform (www.benchling.com) (see Table S2), and inserted in pSpCas9(BB)-2A-GFP (PX458) (\#48138, Addgene) and pU6-(BbsI) CBh-Cas9-T2A-mCherry (\#64324, Addgene) plasmids digested with $B b s$. Cells were plated in six-well plate and transfected with $500 \mathrm{ng}$ of each plasmid using Lipofectamine 3000 (Life Technologies, Thermo Scientific, CA, USA) according to manufacturers' instruction. After $72 \mathrm{~h}$, cells were sorted in a FACSAria II Cell Sorter (BD Biosciences, CA, USA) using an $85 \mu \mathrm{m}$ nozzle and the laser lines $488 \mathrm{~nm}$ and $561 \mathrm{~nm}$. Cells were gated by forward scatter area (FSC-A) vs. side scatter area and FSC-A vs. FSC-width plot to exclude dead cells and doublets/ clumps, respectively. The positive gates were established based on autofluorescence control. GFP/mCherry-positive cells were either collected into a 24-well plate to establish polyclonal cell cultures or collected directly for qPCR analysis. DNA deletions were validated immediately after FACS (1000 cells) or upon the establishment of 
polyclonal cell cultures. DNA was extracted by boiling the samples in Tris- $\mathrm{HCl} \mathrm{pH} 8.0$ for $15 \mathrm{~min}$, incubating with $1 \mu \mathrm{g} / \mu \mathrm{l}$ proteinase $\mathrm{K}$ at $56{ }^{\circ} \mathrm{C}$ for $30 \mathrm{~min}$, and inactivating at $95{ }^{\circ} \mathrm{C}$ for $5 \mathrm{~min}$. Genotyping was performed with iMAX II DNA Polymerase (High Fidelity) (iNtRON Biotechnology, South Korea) according to manufacturers' instructions. The primers used are listed in Table S3.

\section{Time-lapse live-cell imaging}

Cells were plated in four-well or eight-well $\mu$-slides (Ibidi, Germany). Time-lapse images were either acquired in a Leica DMI6000b (Leica Microsystems, Germany) or in a Zeiss Axiovert $200 \mathrm{M}$ (Carl Zeiss, Oberkochen, Germany) inverted microscope. All experiments were done under controlled temperature, atmosphere, and humidity. Neighbor fields were imaged every 5-10 min for $48-72 \mathrm{~h}$. Images were then analyzed in ImageJ/Fiji software.

\section{Western blotting}

Western blotting was performed as previously described in ref. ${ }^{27}$.

\section{Apoptosis assay}

Apoptosis was assessed by flow cytometry using the $\mathrm{BD}$ Accuri C6 (BD Biosciences, CA, USA) and the (FITC)conjugated Annexin V/Apoptosis detection kit (BioLegend, Inc. San Diego, CA, USA). Data were analyzed using the FlowJo v10 software and the gates defined accordingly to the autofluorescence control.

\section{Immunostaining}

Cells were fixed in 4\% paraformaldehyde in PBS for $20 \mathrm{~min}$, permeabilized with $0.3 \%$ Triton-X100 in PBS for $7 \mathrm{~min}$, blocked in $10 \% \mathrm{FBS}$, and incubated overnight at $4{ }^{\circ} \mathrm{C}$ with mouse anti-Flag M2 (Sigma-Aldrich, MO, USA) diluted in 5\%FBS/PBS. Cells were then incubated for $1 \mathrm{~h}$ at room temperature with the secondary antibody AlexaFluor-568 (Life Technologies, CA, USA), and DNA was counterstained with $1 \mu \mathrm{g} / \mathrm{ml}$ DAPI (Sigma-Aldrich, MO, USA). Images were acquired in a Zeiss AxioImager Z1 (Carl Zeiss, Oberkochen, Germany) and analyzed using ImageJ/Fiji software.

\section{Quantitative real-time PCR}

Total RNA was extracted using Quick-RNA Microprep Kit (Zymo Research, CA, USA). cDNA synthesis was performed using NZY First-Strand cDNA Synthesis Kit (NZYtech, Portugal). qPCR analysis was performed in a CFX384 Touch Real-Time PCR Detection System using iTaq Universal SYBR Green Supermix accordingly to manufacturers' instruction, and data analyzed using the CFX Maestro Software (all from Bio-Rad Laboratories, CA, USA). The $2^{-\Delta \mathrm{Ct}}$ or $2^{-\Delta \Delta \mathrm{Ct}}$ methods were used to quantify the transcript levels against three housekeeping genes (GAPDH, HPRT1, TBP). The primers used are listed in Table S4.

\section{Cell cycle profiling}

Cells were harvested, centrifuged at $1500 \mathrm{rpm}$ for $5 \mathrm{~min}$, and rinsed in ice-cold PBS. After centrifugation, cells were resuspended in $500 \mu \mathrm{L}$ of ice-cold PBS. An equal volume of $75 \%$ cold ethanol was added drop-by-drop, while gently vortexing. Samples were chilled on ice for $30 \mathrm{~min}$. Fixed cells were centrifuged at $2000 \mathrm{rpm}$ for $5 \mathrm{~min}$, resuspended in PBS, and incubated with RNase A for $3 \mathrm{~h}$ at $37{ }^{\circ} \mathrm{C}$. Propidium iodide was used at $50 \mu \mathrm{L} / \mathrm{mL}$ for DNA counterstaining. Cells were filtered and the cell cycle profile was assessed by flow cytometry using the BD Accuri C6 (BD Biosciences, CA, USA). Data were analyzed using the FlowJo v10 software.

\section{Bisulphite sequencing}

The EpiTect Bisulphite Kit (Qiagen, DE) was used accordingly to the manufacturers' instructions. The primers used are listed in Table S5.

\section{Luciferase activity assays}

The $B M F$ promoter and CRE\#3 regions were amplified from HDF N genomic DNA by PCR. The $B M F$ promoter amplicon was cloned into pNL1.1 plasmid [Nluc] (Promega \#N1441, WI, USA) to generate Nluc/BMF. The CRE\#3 amplicon was inserted into pCR8/GW/TOPO vector and then cloned into pGL4.23-GW plasmid (Addgene \#60323) via Gateway cloning. siNeg- and siFOXM1-depleted MCF-7 cells were used for the luciferase reporter assays. For the promoter-reporter assay, cells were transfected with $3 \mu \mathrm{g}$ of pGL4.54 [luc2/TK] (Promega \#E5061, WI, USA) and 300 ng of either pNL1.1 (negative control) or NLuc/BMF using Lipofectamine 3000 (Life Technologies, Thermo Scientific, CA, USA) accordingly to manufacturers' instruction. For the enhancer reporter assay, cells were transfected with $30 \mathrm{ng}$ of Nluc and $3 \mu \mathrm{g}$ of either pGL4.23-GW (negative control), luc2/TKp (positive control), or luc2/CRE\#3 plasmid. pGL4.54 [luc2/TK] and pNL1.1 [Nluc] plasmids were used as transfection controls in the promoter and enhancer assays, respectively. Data were acquired using the Nano-Glo® Dual-Luciferase ${ }^{\circledR}$ Reporter Assay System kit (Promega, WI, USA) in a Synergy ${ }^{\mathrm{TM}} 2$ Multi-Mode Microplate Reader (BioTek, VT, USA). The luciferase activity was normalized to the activity of the transfection control and is presented as a relative response ratio, in which the responses to the empty vector and positive control vector were normalized to 0 and 1 , respectively.

\section{C-sequencing}

$10^{6}$ HDF $\mathrm{N}$ cells were used for $4 \mathrm{C}$-seq analysis as previously described ${ }^{50}$ with minor adaptations. Restriction digestions were done with the enzymes DpnII and Csp6I. 
The final template was purified using an Amicon Ultra-15 Centrifugal Filter Device (Millipore, MA, USA). Two libraries were independently prepared with the Expand Long Template polymerase (Roche, $\mathrm{CH}$ ) using primers with adaptors (Table S6). The resulting 4C4C libraries were purified with the QIAquick PCR Purification kit (QIAGEN, DE) followed by the Agencourt AMPure XP reagent (Beckman Coulter, CA, USA). Libraries were sequenced on an Ion S5XL System (Ion Torrent, Thermo Fisher Scientific, MA, USA). Processing and alignment of the sequencing data to the human genome (hg38) were performed as previously described ${ }^{51}$.

\section{Association of drug sensitivities with gene expression levels}

The mRNA and protein expression levels were obtained from CCLE mRNA expression (11/2020 DepMap release; CCLE_Expressionl.Full.csv) and CCLE proteomics (01/ 2020 release; protein_quant_current_normalized.csv) data sets, respectively. The drug sensitivity levels were obtained from the CTRP CTD ${ }^{2}$ data set (12/2015 DepMap release; CTRPv2.0_2015_ctd2_ExpandedDataset.zip). Spearman's correlation coefficient values and linear regression pvalues were calculated using the default association analysis incorporated in the DepMap portal (https://depmap. org/portal/interactive/). The mRNA expression levels of clinical samples were obtained from the Gene Expression Omnibus portal (accession number GSE22358) ${ }^{40}$. The relative expression levels of $B M F$ (probe ID: 37059) and $B U B 1 B$ (probe ID: 20572) were compared between the tumors with the complete or near-complete response and those with no response to a combination of docetaxel and capecitabine, as was previously done for other genes ${ }^{44}$. The statistical analysis between the groups was performed using a two-tailed Mann-Whitney test, in GraphPad Prism 9.0 (GraphPad, CA, USA).

\section{Statistical analysis}

Statistical analysis was performed using GraphPad Prism 8.0 (except association of drug sensitivities with gene expression levels analysis). Sample sizes and statistical tests are indicated in the figure legends for each experiment. Parametric and non-parametric tests were applied adequately. For pairwise comparisons, either a two-tailed $t$ test, two-tailed Mann-Whitney, or two-tailed $\chi^{2}$-square was used, otherwise, two-tailed one-way analysis of variance was applied followed by a post hoc for multiple comparisons test. ns, $p>0.05, " p \leq 0.05,{ }^{* * *} p \leq 0.01,{ }^{* * *} p \leq$ 0.001 , and ${ }^{* * * * *} p \leq 0.0001$. Values are shown as mean \pm S.D.

\section{Acknowledgements}

We acknowledge the technical support of the i3S Scientific Platforms: Advanced Light Microscopy (P. Sampaio), Biosciences Screening (A. Maia) (which are members of the Portuguese Platform of Biolmaging, PPBI-POCl-010145-FEDER-022122) and Genomics (M. Rocha). We thank M. Galhardo for processing and alignment of the $4 C$-seq sequencing data, R. Ribeiro for pLVX$B U B 1 B$ plasmid, and all members in the laboratory for fruitful discussions.

\section{Author details}

${ }^{1}$ i3S - Instituto de Investigação e Inovação em Saúde, Universidade do Porto, 4200-135 Porto, Portugal. ${ }^{2}$ Aging and Aneuploidy Group, IBMC - Instituto de Biologia Molecular e Celular, Universidade do Porto, 4200-135 Porto, Portugal. ${ }^{3}$ Programa doutoral em Biologia Molecular e Celular, Instituto de Ciências Biomédicas Abel Salazar, Universidade do Porto, 4050-313 Porto, Portugal. ${ }^{4}$ Vertebrate Development and Regeneration Group, IBMC - Instituto de Biologia Molecular e Celular, Universidade do Porto, 4200-135 Porto, Portugal. ${ }^{5}$ Graduate Program in Areas of Basic and Applied Biology (GABBA), Instituto de Ciências Biomédicas Abel Salazar (ICBAS), Universidade do Porto, 4050-313 Porto, Portugal. 'Department of Human Molecular Genetics \& Biochemistry, Faculty of Medicine, Tel Aviv University, Tel Aviv, Israel

\section{Author contributions}

Study concept and design and writing-original draft: S.V. and E.L.; development of methodology, data interpretation, and writing-review and editing: all authors; data acquisition and statistical analysis: S.V., F.F., J.M., and G.L.; resources: J.B., U. B.-D., and E.L.; data curation: S.V. and F.F.; supervision, project administration, and funding acquisition: E.L. All authors read and approved the final paper.

\section{Funding}

This work was supported by: FEDER (Fundo Europeu de Desenvolvimento Regional) funds through the COMPETE 2020 - Operational Programme for Competitiveness and Internationalization (POCI), Portugal 2020 and by Portuguese funds through FCT (Fundação para a Ciência e a Tecnologia) in the framework of the project POCI-01-0145-FEDER-031120 (PTDC/BIA-CEL/31120/ 2017); and POCI-01-0145-FEDER-007274 i3S framework project co-funded by COMPETE 2020/ PORTUGAL 2020 through FEDER. S.V. and F.F. were supported by FCT fellowships SFRH/BD/125017/2016 and PD/BD/105745/2014. E.L. was supported by an FCT Investigator Grant (IF/00916/2014). U.B-D. and G.L. were supported by the Azrieli Faculty Fellowship (to U.D.-D.) and the DoD CDMRP Career Development Award (CA191138 to U.B.-D.). J.B. was supported by an FCT Investigator Grant (CEECIND/03482/2018) and the European Research

Council (ERC) under the European Union's Horizon 2020 research and innovation programme (ERC-2015-StG-680156-ZPR).

\section{Data and code availability}

The 4C-seq data sets for this publication were deposited in the European Nucleotide Archive (ENA) at EMBL-EBI under the accession number PRJEB44770 (https://www.ebi.ac.uk/ena/browser/view/PRJEB44770).

Ethics statement

The authors declare that there are no ethical issues.

Conflict of interest

The authors declare no competing interests.

\section{Publisher's note}

Springer Nature remains neutral with regard to jurisdictional claims in published maps and institutional affiliations.

Supplementary information The online version contains supplementary material available at https://doi.org/10.1038/s41419-021-03822-5.

Received: 8 February 2021 Revised: 5 May 2021 Accepted: 6 May 2021 Published online: 25 May 2021

\section{References}

1. Jordan, M. A. \& Wilson, L. Microtubules as a target for anticancer drugs. Nat. Rev. Cancer 4, 253-265 (2004).

2. Weaver, B. A. A. \& Cleveland, D. W. Decoding the links between mitosis, cancer, and chemotherapy: the mitotic checkpoint, adaptation, and cell death. Cancer Cell 8, 7-12 (2005). 
3. Gascoigne, K. E. \& Taylor, S. S. Cancer cells display profound intra- and interline variation following prolonged exposure to antimitotic drugs. Cancer Cell $\mathbf{1 4}$ 111-122 (2008).

4. Laoukili, J. et al. FoxM1 is required for execution of the mitotic programme and chromosome stability. Nat. Cell Biol. 7, 126-136 (2005). 2005.

5. Fischer, M. \& Müller, G. A. Cell cycle transcription control: DREAM/MuvB and RB-E2F complexes. Crit. Rev. Biochem. Mol. Biol. 52, 638-662 (2017).

6. Pilarsky, C., Wenzig, M., Specht, T., Saeger, H. D. \& Grützmann, R. Identification and validation of commonly overexpressed genes in solid tumors by comparison of microarray data. Neoplasia 6, 744-750 (2004).

7. Gentles, A. J. et al. The prognostic landscape of genes and infiltrating immune cells across human cancers. Nat. Med. 21, 938-945 (2015).

8. Arceci, A. et al. FOXM1 deubiquitination by USP21 regulates cell cycle progression and paclitaxel sensitivity in basal-like breast cancer. Cell Rep. 26, 3076-3086.e6 (2019).

9. Carr, J. R., Park, H. J., Wang, Z., Kiefer, M. M. \& Raychaudhuri, P. FoxM1 mediates resistance to herceptin and paclitaxel. Cancer Res. 70, 5054-5063 (2010).

10. Hou, Y. et al. The FOXM1-ABCC5 axis contributes to paclitaxel resistance in nasopharyngeal carcinoma cells. Cell Death Dis. 8, e2659-e2659 (2017).

11. Khongkow, P. et al. Paclitaxel targets FOXM1 to regulate KIF20A in mitotic catastrophe and breast cancer paclitaxel resistance. Oncogene 35, 990-1002 (2016).

12. Zhao, F. et al. Overexpression of forkhead box protein M1 (FOXM1) in ovarian cancer correlates with poor patient survival and contributes to paclitaxel resistance. PLOS ONE 9, e113478 (2014).

13. Yang, N. et al. Aurora kinase A stabilizes FOXM1 to enhance paclitaxel resistance in triple-negative breast cancer. J. Cell Mol. Med. 23, 6442-6453 (2019).

14. Chiu, W. T. et al. FOXM1 confers to epithelial-mesenchymal transition, stemness and chemoresistance in epithelial ovarian carcinoma cells. Oncotarget 6, 2349-2365 (2015).

15. Deschesnes, R. G. et al. Microtubule-destabilizing agents induce focal adhesion structure disorganization and anoikis in cancer cells. J. Pharm. Exp. Ther. $\mathbf{3 2 0}$ 853-864 (2007)

16. Tang, M. K. S., Zhou, H. Y., Yam, J. W. P. \& Wong, A. S. T. c-Met overexpression contributes to the acquired apoptotic resistance of nonadherent ovarian cancer cells through a cross talk mediated by phosphatidylinositol 3-kinase and extracellular signal-regulated kinase 1/2. Neoplasia 12, 128-138 (2010).

17. Tamura, D. et al. Slug increases sensitivity to tubulin-binding agents via the downregulation of $\beta I I I$ and $\beta I V a-t u b u l i n$ in lung cancer cells. Cancer Med. 2 144-154 (2013).

18. Chen, J., Gao, F. \& Liu, N. L1CAM promotes epithelial to mesenchymal transition and formation of cancer initiating cells in human endometrial cancer. Exp. Ther. Med. 15, 2792-2797 (2018).

19. Schmelzle, T. et al. Functional role and oncogene-regulated expression of the $\mathrm{BH} 3$-only factor Bmf in mammary epithelial anoikis and morphogenesis. Proc. Natl. Acad. Sci. USA 104, 3787-3792 (2007).

20. Nagaprashantha, L. D., Vatsyayan, R., Lelsani, P. C. R., Awasthi, S. \& Singhal, S. S. The sensors and regulators of cell-matrix surveillance in anoikis resistance of tumors. Int J. Cancer 128, 743-752 (2011).

21. Hornsveld, M. et al. Restraining FOXO3-dependent transcriptional BMF activation underpins tumour growth and metastasis of E-cadherin-negative breast cancer. Cell Death Differ. 23, 1483-1492 (2016).

22. Hausmann, M. et al. BCL-2 modifying factor (BMF) is a central regulator of anoikis in human intestinal epithelial cells. J. Biol. Chem. 286, 26533-26540 (2011).

23. Girnius, N. \& Davis, R. J. JNK promotes epithelial cell anoikis by transcriptional and post-translational regulation of BH3-only proteins. Cell Rep. 21, 1910-1921 (2017).

24. Ye K., et al. Characterization of an alternative BAK-binding site for BH3 peptides. Nat. Commun. 11, 3301 (2020).

25. Singh, P. K. et al. Dynein light chain binding determines complex formation and posttranslational stability of the BCl-2 family members Bmf and Bim. Cell Death Differ. 27, 434-450 (2019).

26. Puthalakath, $\mathrm{H}$. et al. Bmf: $\mathrm{A}$ proapoptotic $\mathrm{BH}$-only protein regulated by interaction with the myosin $\mathrm{V}$ actin motor complex, activated by anoikis. Science 293, 1829-1832 (2001).
27. Macedo, J. C. et al. FoxM1 repression during human aging leads to mitotic decline and aneuploidy-driven full senescence. Nat. Commun. 9, 2834 (2018).

28. Sun L., et al. The FOXM1 inhibitor RCM-1 suppresses goblet cell metaplasia and prevents IL-13 and STAT6 signaling in allergen-exposed mice. Sci Signal 10, eaai8583 (2017).

29. Gormally, M. V. et al. Suppression of the FOXM1 transcriptional programme via novel small molecule inhibition. Nat. Commun. 5, 5165 (2014).

30. Singh, R., Letai, A. \& Sarosiek, K. Regulation of apoptosis in health and disease: the balancing act of BCL-2 family proteins. Nat. Rev. Mol. Cell Biol. 20, 175-193 (2019).

31. Shao, Y. \& Aplin, A. E. Akt3-mediated resistance to apoptosis in B-RAF-targeted melanoma cells. Cancer Res. 70, 6670-6681 (2010).

32. Sanders, D. A., Ross-Innes, C. S., Beraldi, D., Carroll, J. S. \& Balasubramanian, S. Genome-wide mapping of FOXM1 binding reveals co-binding with estrogen receptor alpha in breast cancer cells. Genome Biol. 14, R6 (2013).

33. Dunham, I. et al. An integrated encyclopedia of DNA elements in the human genome. Nature 489, 57-74 (2012).

34. Carr, J. R. et al. FoxM1 regulates mammary luminal cell fate. Cell Rep. 1, 715-729 (2012)

35. Yao, S., Fan, L. Y. N. \& Lam, E. W. F. The FOXO3-FOXM1 axis: a key cancer drug target and a modulator of cancer drug resistance. Semin. Cancer Biol. 50, 77-89 (2018)

36. Montavon, T. et al. A regulatory archipelago controls hox genes transcription in digits. Cell 147, 1132-1145 (2011).

37. Risinger, A. L., Dybdal-Hargreaves, N. F. \& Mooberry, S. L. Breast cancer cell lines exhibit differential sensitivities to microtubule-targeting drugs independent of doubling time. Anticancer Res. 35, 5845-5850 (2015).

38. Barretina, J. et al. The Cancer Cell Line Encyclopedia enables predictive modelling of anticancer drug sensitivity. Nature 483, 603-607 (2012).

39. Seashore-Ludlow, B. et al. Harnessing connectivity in a large-scale smallmolecule sensitivity dataset. Cancer Discov. 5, 1210-1223 (2015).

40. Glück, S. et al. TP53 genomics predict higher clinical and pathologic tumor response in operable early-stage breast cancer treated with docetaxel-capecitabine \pm Trastuzumab. Breast Cancer Res. Treat. 132, $781-791$ (2012).

41. Yang, J. et al. Pathological Ace2-to-Ace enzyme switch in the stressed heart is transcriptionally controlled by the endothelial Brg1-FoxM1 complex. Proc. Natl. Acad. Sci. USA 113, E5628-E5635 (2016).

42. Sloss, O., Topham, C., Diez, M. \& Taylor, S. Mcl-1 dynamics influence mitotic slippage and death in mitosis. Oncotarget 7, 5176-5192 (2016).

43. Allan, L. A. \& Clarke, P. R. Phosphorylation of caspase-9 by CDK1/cyclin B1 protects mitotic cells against apoptosis. Mol. Cell 26, 301-310 (2007).

44. Topham, C. et al. MYC is a major determinant of mitotic cell fate. Cancer Cell 28, 129-140 (2015).

45. Zierhut, $C$. et al. The cytoplasmic DNA sensor cGAS promotes mitotic cell death. Cell 178, 302-315.e23 (2019).

46. Krishnan, A. et al. Oncogenic actions of SKP2 involves deregulation of CDK1 turnover mediated by FOXM1. J. Cell Biochem 118, 797-807 (2017).

47. Major, M. L., Lepe, R. \& Costa, R. H. Forkhead box M1B transcriptional activity requires binding of Cdk-cyclin complexes for phosphorylation-dependent recruitment of p300/CBP coactivators. Mol. Cell Biol. 24, 2649-2661 (2004).

48. Pan $H_{\text {., }}$ Zhu $Y$., Wei W., Shao S., Rui X. Transcription factor FoxM1 is the downstream target of c-Myc and contributes to the development of prostate cancer. World. J. Surg. Oncol. 16, 59 (2018).

49. Wierstra, I. \& Alves, J. FOXM1C transactivates the human c-myc promoter directly via the two TATA boxes P1 and P2. FEBS J. 273, 4645-4667 (2006).

50. Van De Werken H. J. G., et al. 4C technology: protocols and data analysis. In: Methods in Enzymology. Academic Press Inc., pp 89-112 (2012).

51. Eufrásio, A. et al. In vivo reporter assays uncover changes in enhancer activity caused by type 2 diabetes associated SNPs. Diabetes 69, db191049 (2020).

52. Huang, D. W., Sherman, B. T. \& Lempicki, R. A. Systematic and integrative analysis of large gene lists using DAVID bioinformatics resources. Nat. Protoc. 4, 44-57 (2009). 